\title{
COST-EFFECTIVENESS OF HUMAN LEUKOCYTE ANTIGEN MATCHING IN PENETRATING KERATOPLASTY
}

Michael Bäumler, Leonie Sundmacher

Department of Health Service Management, Ludwig-Maximilians-University
Thomas Reinhard, Daniel Böhringer

Eye Clinic of the University Medical Center, University of Freiburg

Background: The matching of favorable human leukocyte antigen (HLA) combinations is rarely performed in penetrating keratoplasty procedures for primary prophylaxis of immune reactions. However, clinical studies suggest that the incidence of graft rejection decreases substantially when patients receive favorably matched grafts.

Objective: The aim of this study was to assess the cost-effectiveness of HLA matching for patients undergoing penetrating keratoplasty in everyday clinical practice.

Methods: In the absence of a randomized controlled clinical trial, we used administrative data from the Freiburg University Eye Hospital in Germany. Our study population consisted of all patients who underwent their first keratoplasty between 11/2003 and 01/2010 and for whom information on HLA histocompatibility was available. We used propensity score matching to estimate a causal effect of favorable HLA matching, parametric survival regression techniques to predict graft survival and expert opinion to model incremental cost for HLA matching. Because the availability of favorable HLA histocompatibility ultimately depends on the patients' HLA phenotype, we modeled the incremental cost-effectiveness ratio (ICER) as a function of the probability that a patient will receive a favorably matched HLA, and used expert opinion to set a point estimate.

Results: We predicted that corneal grafts with favorable HLA matching were associated with improved rejection-free graft survival time (more than 1,000 days). We estimated the incremental cost of HLA matching at EUR 1,200 and the ICER at EUR 4.62 per additional day of graft survival.

Conclusions: The ICER of HLA matching is acceptable, given the high cost of alternative treatment and the shortage of corneal donors in Germany.

Keywords: Penetrating keratoplasty, HLA matching, corneal graff, cost-effectiveness analysis, administrative data

The economic and social burden of blindness is immense. In Germany alone, the direct cost associated with visual disturbances and blindness (ICD-10 H53-54) was €359 million in 2008 , up from $€ 280$ million in 2002 (1). Apart from its economic cost, blindness also leads to a dramatic decrease in quality of life (2). Severe corneal disease, one of the major causes of blindness, can be treated with a procedure known as penetrating keratoplasty, which entails removing a patient's diseased cornea and replacing it with donated corneal tissue (3).

Based on data from the Australian Corneal Graft Registry, Coster and Williams (4) found that despite the use of topical steroids, the cumulative survival rate for corneal grafts 5 years after implantation was less than 70 percent. This graft rejection leads to a substantial worsening of vision. Systemic immunoprophylaxis can be used to decrease rejection rates, but the side effects of immunosuppressants may both outweigh the benefits associated with longer graft survival (5) and cause high cost for the healthcare system, with a daily defined dose (DDD) costing nearly EUR 13. Re-interventions after rejection represent a further drain on resources, as some patients must undergo repeat keratoplasties at high cost. Finally, supply of grafts is limited because it is based on the availability of donor tissue. Germany's shortage of corneal grafts is apparent in the number of patients on the transplant waiting list: as of 31 December 2008, 2,281

We thank Mr. Matthew Gaskins and Ms. Kate O'Neil for language editing and helpful comments. patients were awaiting corneal transplant (data made available upon request from the German Keratoplasty Registry, which is managed by the University of Saarland on behalf of the Cornea Section of the German Ophthalmological Society). Although artificial corneas, known as keratoprostheses, may present an alternative to grafts at some point in the future, their use to date has been limited to a small group of patients (6). Therefore, cost-effective measures to reduce the number of graft rejections from penetrating keratoplasty remain sorely needed.

Several studies have shown that favorable matching for human leukocyte antigens (HLA) between donors and recipients has a positive impact on rejection-free graft survival. HLA Matchmaker is a novel "in silico" approach to HLA matching developed by Duquesnoy (7), which uses a comprehensive database to predict the set of antibody-accessible epitopes from any HLA phenotype. This method allows a more sophisticated approach to matching than conventional uniform "stacking" of all foreign alleles: multiple HLA-mismatches commonly share antibody epitopes and thus do not count fully as suggested by conventional matching. The observation that certain "taboo" mismatches are more immunogeneic than others (8) supports this concept. The HLA Matchmaker technique has been developed and successfully applied to penetrating keratoplasty by Böhringer et al. (9), and the technique has been associated with a substantial prolongation of rejection-free graft survival (a detailed description of the procedure is presented in Böhringer 
et al.) (9). Based on an exploratory and a confirmational cohort, the authors classified the histocompatibility patterns into three groups: (a) favorable match: fewer than 10 mismatched HLA-class I eplets combined with a beneficial HLA-class I and class II matching balance; (b) neutral match: fewer than 10 mismatched HLA-class I eplets or an unfavorable HLA-class I and class II matching balance; (c) unfavorable match: more than 10 mismatched HLA-class I eplets and an unfavorable HLA-class I and class II matching balance.

This classification was highly predictive of immune reactions in both exploratory and confirmational datasets and seems to be very promising.

Although implanting favorably matched grafts seems to offer an advantage over assigning grafts without regard to HLA phenotype, the European Eyebank Association (EEBA) surveys suggest that the percentage of HLA-matched transplantations in Europe may be as low as 2 percent. Economic factors might be responsible for this low rate. As soon as any hospital decides to offer HLA matching, all patients and available grafts must be HLA-typed. However, not all patients who undergo HLA matching will actually receive favorably matched grafts within a reasonable timeframe. The availability of histocompatible grafts ultimately depends on the population frequencies of the individual HLA phenotypes. Even if HLA matching is part of routine care, rare phenotypes are not likely to receive a matched graft due to a prohibitively small pool of HLAtyped compatible donors (10). Thus, only the share of patients who receive a favorably matched graft will benefit from HLA matching. Moreover, HLA matching offers no additional benefit to those patients who might in any case receive a favorably matched graft just by chance. Thus, although HLA matching does incur an incremental cost, there is enormous uncertainty about the incremental effectiveness of providing HLA matching in everyday clinical practice. For this reason, an economic evaluation of HLA matching is inherently different from evaluation of other technologies.

To provide a framework for evidence-based decisions regarding the provision of HLA matching in routine care for all patients, decision makers therefore need information about (a) the incremental cost of HLA matching and (b) the incremental effects of HLA matching on graft survival. The latter is closely related to the proportion of patients who will-after HLA matching - be able to receive a favorably matched graft they would not have received by chance.

Although the matching approach of Böhringer et al. (9) seems to have a significant effect on graft survival, no economic study to date has evaluated this procedure. We aim to fill this gap with the present analysis. First, we aim to give an estimate for the incremental cost of HLA matching in penetrating keratoplasties. Second, we intend to measure the incremental effect of favorably matched grafts in terms of rejection-free graft survival. As the matching technique by Böhringer et al. has only been developed recently, data from a randomized controlled clinical trial
(RCT) that compares patients who have undergone HLA matching with patients who have not undergone HLA matching are not yet available. To estimate a causal effect in a non-RCT setting, we use a state-of-the-art propensity score matching approach. To deal with right-censored data, we use parametric regression techniques to extrapolate median survival times. Based on the incremental effect of favorable matches on rejection-free graft survival, we suggest an approach which accounts for the uncertainty regarding the availability of favorably matched grafts and give an estimate for an ICER based on expert opinion.

This study is structured as follows: In the Methods section, we present the methods we used to address these research questions; in the Results section, we discuss our results; and in the Discussion section, we present conclusions based on our findings.

\section{METHODS}

\section{Setting and Data}

In the absence of data from a randomized controlled trial or prospective data, we used retrospective clinical data derived from all patients who underwent a penetrating keratoplasty at the Freiburg University Eye Hospital between 1 November 2003 and 31 January 2010. During this period, a total of 1,495 penetrating keratoplasties were performed. For the purposes of scientific research, the hospital routinely typed the HLA of all patients and most of the grafts, even when an HLA-matching graft was not actively sought. However, prospective conventional HLA matching was only performed on a subset of patients who were classified as "high-risk," as described below. We excluded all patients for whom we did not have information on the patient's and grafts' HLA. We also excluded all patients who had undergone repeat keratoplasties because including them in our analysis would have complicated the propensity score matching procedure. HLA histocompatibility was classified retrospectively based on HLA information from patient and donor. The novel classification scheme developed by Böhringer et al. (9) was used and we distinguished between three types of HLA histocompatability based on HLAMatchmaker eplets: (a) favorable, (b) neutral, and (c) unfavorable. Both neutral and unfavorable matches were defined as a reference to assess the incremental benefit of favorable matching on rejection-free graft survival. All patients consented to scientific analysis of the routine data, and permission was obtained from the ethics committee of the Freiburg University Hospital.

All data were obtained by reviewing patient charts, which included information on the age and gender of each patient, as well as their indication for penetrating keratoplasty. Unfortunately, cost data was not available in the patient records. In the present study, we distinguished between indications with (i) a high or (ii) a low number of patients. The first group consisted of patients with keratoconus, Fuchs' endothelial dystrophy, avascular corneal scars, or degeneration of the corneal endothelium 
(bullous keratopathy). The second group consisted of patients with any other indication. Graft rejections were defined as endothelial precipitates, newly diagnosed corneal edema (not attributable to increased intraocular pressure), nummular stromal infiltrates in the graft or epithelial rejection lines. The hospital also classified implants as low-risk or high-risk, according to their estimated risk of rejection based on whether the procedures involved repeat keratoplasties, vascularized corneas, or surface disorders, etc. $(11 ; 12)$. Graft failures that did not result from graft rejections were not used as endpoints. We created a variable which assumed a value of one if a graft rejection occurred during the study period, or a value of zero if no graft rejection occurred during the study period. Event-free time (i.e., time without rejection) was calculated in days and defined as the time until the graft was rejected or the patient was seen for the last time. In the latter case, the true survival time of the corneal graft was unknown and the data were considered right-censored.

\section{Propensity Score Matching}

Because we analyzed the administrative data retrospectively, we could not rule out that patients in the favorable matches group differed substantially in terms of characteristics that are associated with the survival time of the graft. To preclude the possibility that increased rejection-free graft survival time was not chiefly attributable to patient characteristics, we used propensity score matching to compare only patients with favorable matches and controls who were homogeneous in terms of their outcome-relevant characteristics (13). There are several economic evaluations based on secondary data that take similar approaches, for example, Bäumler et al. (14). The propensity score $\mathrm{P}_{\mathrm{i}}$ (i.e., the probability of keratoplasty $\mathrm{i}$ being assigned to the favorable match cohort) aimed to control for all characteristics which are known to be associated with graft rejections. This was defined as a function of patient age, patient gender, the risk of graft rejection, and the indication for keratoplasty, as follows: $\mathrm{P}_{\mathrm{i}}(\mathrm{X})=\mathrm{F}$ (age, gender, risk status, indication).

To estimate the propensity score, we used a logistic regression. We subsequently attempted to match patients in the favorable match cohort with controls who had roughly the same propensity scores. There are several methods for matching (for an overview see Austin $(15 ; 16))$. As our principal aim was to reduce heterogeneity between the two groups, we opted for the one-to-one nearest neighbor matching method with replacement and a calipers of width equal to 0.01 . The same control could theoretically be used for several patients in the favorable match cohort. If no suitable control for a patient in the favorable match cohort was found within a caliper of 0.01 around his or her propensity score, this patient was excluded from further analysis to minimize differences between the groups. The width of 0.01 was chosen as it resulted in a good trade-off between heterogeneity and sample size. Standardized Differences (SDF) for each outcome-relevant variable were used to evaluate the goodness-of-fit. SDFs below a threshold of 10 percent suggest that favorable match cohort and control cohort differed only in the HLA-matching classification of the graft (i.e., favorable versus neutral or unfavorable) (16).

\section{Incremental Cost}

In the absence of cost data in the patient records, we modeled cost based on treatment guidelines published by the German Society of Ophthalmologists (7) and expert opinions provided by ophthalmologists of the Freiburg University Eye Hospital. Because our analysis was conducted from the perspective of the payer, we did not consider indirect cost (8). According to these sources, total cost for patients who undergo a penetrating keratoplasty comprise (a) the cost of the graft itself, (b) the cost of hospital admission and the keratoplasty procedure, and (c) the cost of follow-up visits and medication during the follow-up period. The treatment guidelines state that resource consumption during the follow-up period is the same regardless of HLA matching. This also holds for the keratoplasty procedure and hospital admission. Thus, the only incremental cost incurred is in the preparation of the graft. According to expert opinion, the only difference between grafts prepared for HLA matching and randomly selected grafts is that HLA matching involves typing of both patient and donor (i.e., two times the cost for one typing procedure). The cost of matching software is virtually negligible.

\section{Incremental Effect of Favorable Matches on Rejection-Free Graft Survival}

As the patient charts do not provide information on patientrelated outcomes such as utility values, we chose graft survival as an outcome measure, defined as rejection-free graft survival time. First, we used Kaplan-Meier estimates to illustrate rejection-free graft survival time. For the vast majority of datasets included in our analysis, the true survival time of corneal grafts was unknown because rejection did not occur during the study period. Our data was thus heavily right-censored. We therefore relied on a regression-based extrapolation of median rejection-free graft survival time (20), defining the survival time $S_{i}$ of graft $i$ as a function of patient age, gender, risk of graft rejection, indication, and HLA matching classification: $\mathrm{S}_{\mathrm{i}}=\mathrm{F}$ (age, gender, risk status, indication, HLA matching classification).

As a distribution for the survival regression, we used the Weibull distribution which seemed to be suitable for our purposes, as it allows flexibility. Because the decision regarding which distributions to use for the regression model is ultimately arbitrary, (19) different distributions were considered in the sensitivity analyses. Subsequently, we used the estimates from the hazard regression to predict the median rejection-free graft survival time for an average patient with and without favorable HLA matching. 


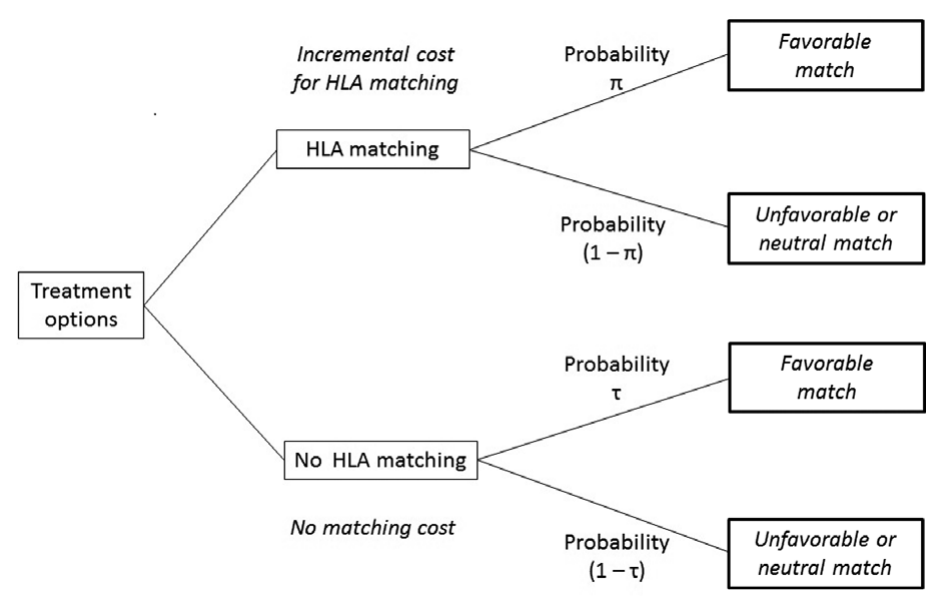

Figure 1. Illustration of treatment options.

Incremental Cost-Effectiveness-Ratio

To provide information about the cost-effectiveness of this procedure, we aimed to calculate an incremental cost-effectiveness ratio (ICER). An ICER is defined as

$$
\mathrm{ICER}=\frac{\Delta \text { Cost }}{\Delta \text { Effectiveness }}
$$

The raw $\mathrm{ICER}_{\mathrm{R}}$ below represents the formula which would have been used had we been able to ascertain that all patients who had undergone HLA matching had received a favorably matched graft and, in addition, had not been able to receive a favorably matched graft without HLA matching:

$$
\operatorname{ICER}_{R}=\frac{\Delta \text { Cost }}{\Delta \text { Survival }}
$$

where $\Delta$ Cost is the cost for HLA typing and $\Delta$ Survival is the incremental rejection-free graft survival of the favorably matched graft. However, in a real-world setting there is uncertainty about the effectiveness of HLA matching because, due to the limited availability of various HLA phenotypes in grafts, only a share of patients will be able to receive a favorably matched graft after HLA matching. In addition, even if the hospital does not use HLA matching and no cost are incurred from HLA typing, a certain proportion of patients will receive a favorably matched graft merely by chance.

To account for this in our economic analysis, we made the following adjustments to the raw ICER. The share of patients receiving a favorably matched graft is represented as $\pi$ $(0 \leq \pi \leq 1)$ and the effectiveness of HLA matching is represented as $\pi$ times the incremental gain in graft survival time, where $\pi$ depends on the availability of HLA phenotypes with a favorable histocompatibility. The share of patients who will receive a favorably matched graft merely by chance is represented by $\tau(0 \leq \tau \leq 1)$. The effectiveness of not conducting HLA matching thus equals $\tau$ times the incremental gain in graft survival time, where $\tau$ is a stochastical function of the availability of matching grafts, which is referred to in the parameter $\pi . \pi$ is thus by definition greater than or equal to the share $\tau$. Figure 1 illustrates these uncertainties in a decision-tree structure.

Following this, the share of patients that does benefit from HLA matching by receiving a favorably matched graft is defined as $(\pi-\tau)$. A corrected ICER which accounts for uncertainty regarding the availability of grafts with a favorable HLA histocompatibility is thus defined as

$$
\operatorname{ICER}_{C}=\frac{\Delta \text { Cost }}{(\pi-\tau) \Delta \text { Survival }}=\frac{1}{(\pi-\tau)} \operatorname{ICER}_{R}
$$

The corrected ICER is therefore a function of the inverse share of patients who receive a favorably matched graft due to HLA matching. In the absence of data that would enable the authors to estimate the parameters $\pi$ and $\tau$, we draw on expert opinion from ophthalmologists to give a point estimate for the ICER.

\section{Sensitivity Analyses}

Apart from uncertainty regarding the proportion of patients $(\pi-\tau)$ that benefit from HLA matching, further uncertainty was present in our results regarding (i) our extrapolation technique and (ii) the cost of the intervention. Therefore, we also performed multivariate sensitivity analyses to test our results' robustness. To account for uncertainty regarding our choice of a Weibull distribution, we extrapolated the incremental survival time using exponential, log-logistic, and log-normal distributions. Given that HLA typing is a mostly automated procedure, we reckoned it was reasonable to assume economies of scale when the number of typed grafts increased. Estimating a lower cost for one typing procedure might therefore more accurately reflect the future cost of such procedures.

\section{RESULTS}

\section{Baseline Characteristics of the Patients}

In our data set of 721 keratoplasties, we identified 118 patients in the favorable match cohort and 603 in the control cohort. The 
Table 1. Characteristics of the Study Population

\begin{tabular}{|c|c|c|c|c|c|c|c|c|}
\hline \multirow[b]{2}{*}{ Variable } & \multicolumn{4}{|c|}{ Before PSM } & \multicolumn{4}{|c|}{ After PSM } \\
\hline & $\begin{array}{c}\text { Control cohort } \\
N=603 \\
\text { Mean } / \%\end{array}$ & $\begin{array}{l}\text { Favorable } \\
\text { match cohort } \\
N=118 \\
\text { Mean / \% }\end{array}$ & $p$-Value & $\begin{array}{c}\text { SDF before } \\
\text { PSM }\end{array}$ & $\begin{array}{c}\text { Control cohort } \\
N=115 \\
\text { Mean / \% }\end{array}$ & $\begin{array}{l}\text { Favorable } \\
\text { match cohort } \\
N=115 \\
\text { Mean } / \%\end{array}$ & $\begin{array}{l}\text { SDF after } \\
\text { PSM }\end{array}$ & $\Delta S D F$ \\
\hline Age & $58.7(19.7)$ & $52.9(18.9)$ & .003 & $29.9 \%$ & $53.2(17.5)$ & 53.3 (18.7) & $0.8 \%$ & $-29.2 \%$ \\
\hline Female patients & $47.8 \%$ & $36.4 \%$ & .024 & $47.0 \%$ & $37.4 \%$ & $37.4 \%$ & $0.0 \%$ & $-47.0 \%$ \\
\hline High-risk patients & $23.9 \%$ & $38.1 \%$ & .001 & $67.7 \%$ & $37.4 \%$ & $39.1 \%$ & $7.4 \%$ & $-60.3 \%$ \\
\hline Common indications & $85.9 \%$ & $79.7 \%$ & .083 & $43.6 \%$ & $80.0 \%$ & $79.1 \%$ & $5.3 \%$ & $-38.3 \%$ \\
\hline
\end{tabular}

Standard deviation in parentheses for continuous variables.

The $p$ values are derived from T-test (continuous variables) and chi-square tests (categorical variables).

SDF $=$ standardized difference.

SDF for most of the variables was rather high. Detailed descriptions of patient baseline characteristics are given in Table 1.

\section{Propensity Score Matching}

To ensure that our propensity score matching would minimize all potential biases, we included a large number of variables and made certain that diverse interactions between the variables were considered in the logistic regression (the results of the regression are available upon request from the authors). Propensity score matching led to a substantial reduction in heterogeneity (see Table 1), with the SDF for all variables remaining below a threshold of 10 percent. The total sample size was reduced to 230 (with 115 patients in each cohort) because we were unable to find comparable controls for three of the original 118 patients in the favorable match cohort.

\section{Cost Analysis}

We assumed a cost of EUR 600 for each typing procedure based on Böhringer et al. (20). As there are virtually no cost differences between matched and randomly selected grafts, and incremental cost is the sum of the cost for typing the graft and the patient, the total incremental cost was estimated at EUR 1,200 .

\section{Rejection-Free Graft Survival Time Prediction}

Figure 2 shows the Kaplan-Meier survival curves. The favorable match cohort is shown in blue and the control cohort in red. Data for a substantial share of patients were right-censored. There were a total of 20 corneal graft rejections in the favorable match cohort and 28 in the control group during the study period. After 776 days, no known graft rejections took place in the favorable match cohort, whereas in the control cohort several graft rejections did occur.
Results from the regression model suggest that favorable HLA matching based on HLA Matchmaker-defined eplets was significantly associated with rejection-free graft survival time $(P=0.04)$. We estimated a survival time of 2,573 days in the favorable match group and 1,444 days in the control group. The incremental effect of receiving a favorable match on rejectionfree graft survival is thus 1,129 days.

\section{Incremental Cost-Effectiveness Ratio and Sensitivity Analyses}

We calculated a raw ICER $_{R}$ of EUR 1.06 for each additional day of graft survival. This raw value represents what the ICER would have been had all patients received a favorably matched graft and the probability of receiving such a graft without HLA matching were zero. The corrected ICER $\mathrm{C}_{\mathrm{C}}$ was thus defined as ICE $R_{C}=\frac{1}{\pi-\tau} 1.06$.

For the parameters $\pi$ and $\tau$, we used estimates that were agreed upon by experts in ophthalmology from the Freiburg University Hospital who have longstanding experience in conducting penetrating keratoplasty and HLA matching. We assumed that the share of patients who received a favorably matched graft by chance (our parameter $\tau$ ) was lower than 10 percent. Experts believe that if patients undergo prospective HLA matching more than one third of the patients will receive a favorably matched graft. For our analysis, therefore, conservative estimates for the parameters $\tau$ and $\pi$ ( $\tau=0.1$ and $\pi=$ 0.33 ) were assumed. Based on these figures, the share of the patients receiving a favorably matched graft due to HLA matching equaled 0.23 , yielding a point estimate of EUR 4.62 for each additional day of graft survival. This result and the distribution of ICER $_{C}$ is shown in Figure 3.

Point estimates for the sensitivity analyses range from EUR 2.10 to EUR 6.71 per additional day of graft survival. Table 2 sums up the results of the sensitivity analyses. 


\section{Kaplan-Meier Survival Curve of the matched population}

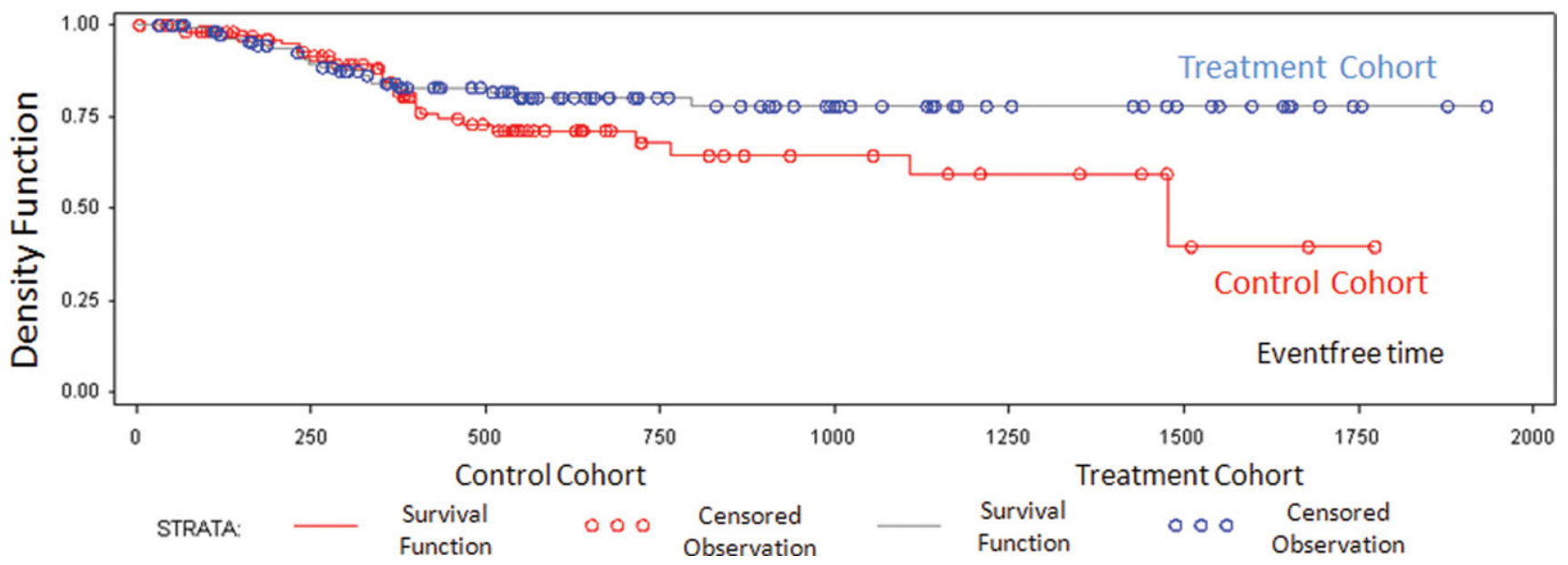

Figure 2. Kaplan-Meier survival curves.

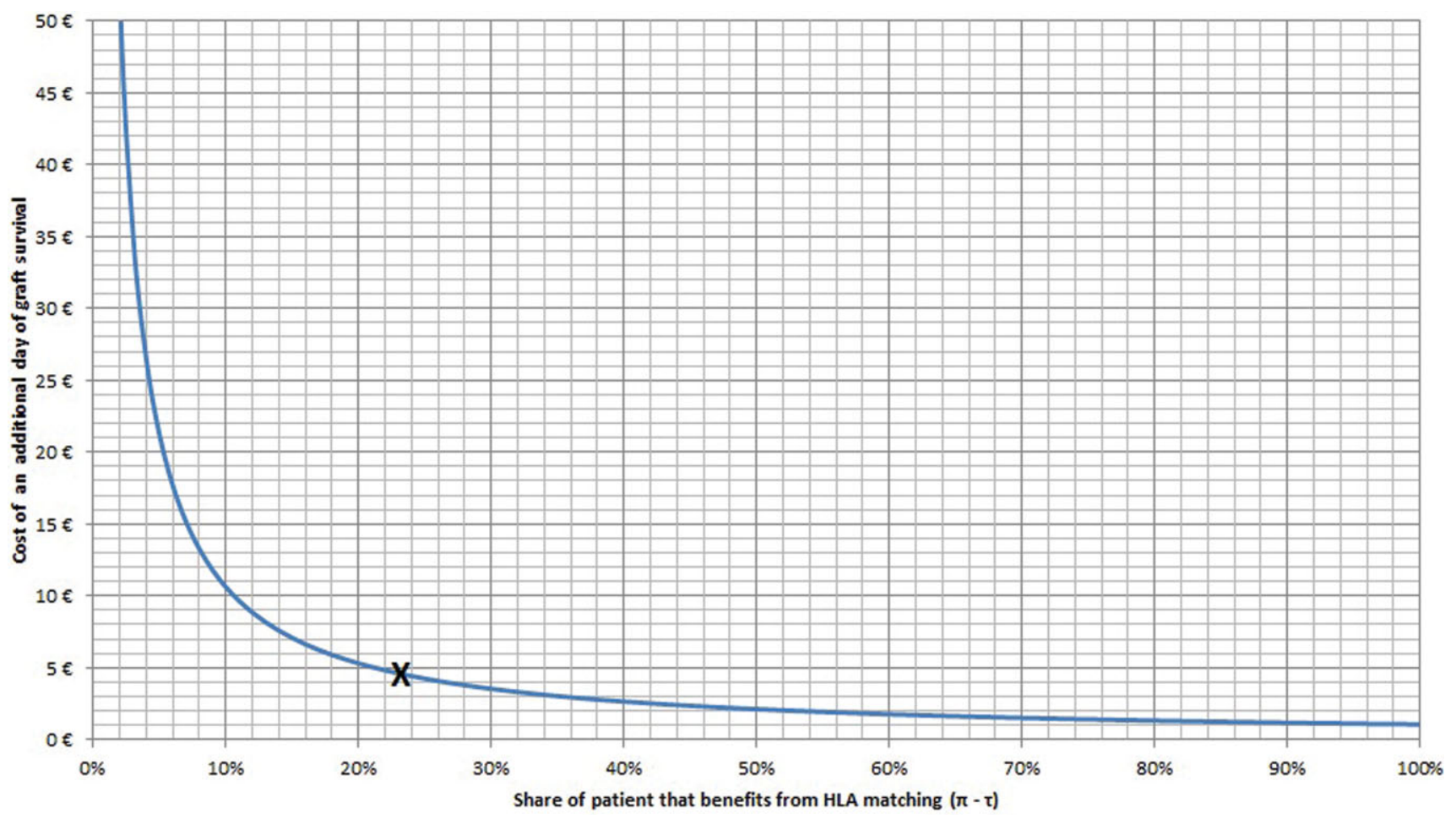

Figure 3. Raw ICER as a function of $(\pi-\tau)$. The point estimate for the ICER is marked with X.

\section{DISCUSSION}

We conducted a retrospective cohort study of patients who had undergone penetrating keratoplasty at the Freiburg University Eye Hospital to assess the incremental cost and effectiveness of favorable HLA matching using HLA Matchmaker-defined eplets. Cost was modeled based on treatment guidelines from a payer's perspective. Effectiveness was defined as rejectionfree survival time of the corneal graft. To our knowledge, our study is the first to investigate the cost and effectiveness of HLA matching in penetrating keratoplasty.

HLA matching is associated with higher cost because both patient and graft must be HLA typed. We assumed an incremental cost of EUR 1,200 for HLA matching (twice the cost of EUR 600 for each typing). However, given that HLA typing is a mostly automated procedure, we consider it reasonable to assume that the typing cost would decrease when further 
Table 2 Results Including Sensitivity Analyses

\begin{tabular}{lccccccccc}
\hline Distribution & \multicolumn{2}{c}{$\begin{array}{c}\text { Weibull } \\
\text { (Baseline analysis) }\end{array}$} & \multicolumn{2}{c}{ Exponential } & Log-log & & Log-normal \\
\hline Incremental cost & $€ 1,200$ & $€ 600$ & $€ 1,200$ & $€ 600$ & $€ 1,200$ & $€ 600$ & $€ 1,200$ & $€ 600$ \\
Incremental survival & $1,129 * *$ & $1,129 * *$ & $1,244^{* *}$ & $1,244^{* *}$ & $970^{*}$ & $970^{*}$ & 777 & 777 \\
Raw ICER & $€ 1.06$ & $€ 0.53$ & $€ 0.96$ & $€ 0.48$ & $€ 1.24$ & $€ 0.62$ & $€ 1.54$ & $€ 0.77$ \\
& per day & per day & per day & per day & per day & per day & per day & per day \\
ICER estimate & $€ 4.62$ & $€ 2.31$ & $€ 4.19$ & $€ 2.10$ & $€ 5.38$ & $€ 2.69$ & $€ 6.71$ & $€ 3.36$ \\
& per day & per day & per day & per day & per day & per day & per day & per day
\end{tabular}

This raw ICER has to be multiplied with the inverse share of patients who receive a favorably matched due to HLA matching.

$* p<0.1$

${ }^{* *} p<00.05$

hospitals implemented HLA matching, based on the principle of economies of scale. We found favorable HLA matching had a significant effect on rejection-free graft survival time. Results were robust to various distributions of the hazard function; only in the log-normal model did favorable matches not have a significant impact on graft survival. We therefore conclude that favorable HLA matching based on HLA Matchmaker-defined eplets is significantly associated with longer rejection-free graft survival time. Böhringer et al. (9), who devised the HLA-matching technique upon which our analysis is based, obtained results comparable to ours, albeit without having performed propensity score matching. We found a raw ICER of EUR 1.06 per additional day of graft survival, which was multiplied by the inverse of the share of patients that benefited from HLA matching. Based on a very conservative expert opinion, we estimated an ICER of 4.62 per additional day of graft survival. Results of the sensitivity analyses ranged from EUR 2.10 to EUR 6.71. Our findings were most sensitive to incremental cost, whereas they are less sensitive to the assumption of various distributions. If all eye banks typed grafts routinely, it would be reasonable to assume that the share of patients that received a favorably matched graft would increase substantially. As a consequence of this, the ICER would decrease.

Our analysis was based on patient claim data. We thus did not have information on patient-reported outcomes. In addition, there are no utility values for patients who underwent a penetrating keratoplasty in the literature. As a result, we could not estimate a cost per quality-adjusted life-year (QALY) ratio and had to rely on surrogate outcomes such as the chosen rejectionfree graft survival that was available in our dataset. In contrast to a cost per QALY ratio, there is no accepted threshold for cost per additional day of graft survival. Subsequently, we cannot judge the absolute superiority of this technique. However, by comparing the cost of alternative treatment strategies, we can evaluate its relative superiority.
An alternative strategy for decreasing the rejection rates of corneal grafts is the intake of cyclosporin A or mycophenolate mofetil $(21 ; 5)$. However, not only do such treatments cause sideeffects harmful to the patient, but cost from a payers' perspective are higher. According to the German pharmaceutical catalogue Rote Liste, both treatments incur a cost of nearly EUR 13 per day. This is substantially higher than our estimated ICER of EUR 4.62 for HLA Matching. Based on our raw ICER, HLA matching is superior if at least 9 percent of the patients receive a favorably matched graft due to HLA matching (where the patient share is $\pi-\tau$ ).

However, even if the share of patients is lower than this percentage, there is a high probability that this technology is costeffective. When conducting an economic evaluation of transplantation techniques, it is important to consider that grafts are inherently different from other medical technologies. The demand for grafts significantly surpasses the number of available donors, creating a shortage of grafts worldwide. However, the high shadow value of grafts resulting from this scarcity cannot be factored into the pricing of grafts in economic evaluations because assigning monetary value to human body parts is widely viewed as unethical. This suggests that we tend to underestimate the value of transplantation techniques that increase (a) the survival time of grafts and thus (b) the number of available grafts within the regulated systems for allocating these grafts. Given that the estimated ICER in our study did not take into account the nonmonetary value of grafts, it is reasonable to assume that favorable HLA matching is cost-effective.

\section{Limitations}

This study has several important limitations. First, our analysis is not based on RCT data but on a retrospective observational study. We used a propensity score matching technique to account for all known characteristics that might be associated with the survival time of the graft, and we included various interactions 
between the variables. Although this led to a balance in both cohorts in terms of observable characteristics, we cannot entirely rule out the presence of unobservable characteristics that might have confounded our results. Second, it is important to keep in mind that we had to extrapolate the rejection-free graft survival time far beyond the last available data point. Several economic evaluations have calculated restricted mean survival time to tackle the problem of censored data and the resulting decrease in the reliability of Kaplan-Meier estimates $(22 ; 23)$. If we had chosen this method, however, our study period would have comprised only 776 days. Considering the average rejectionfree survival times of corneal grafts reported in other studies (e.g., Böhringer et al.) (9), this period would have been too short to evaluate the effects of HLA matching on rejection-free graft survival time. This applies in particular to grafts that do not fail immediately after an endothelial immune reaction. In such cases, the graft clears but suffers a substantial loss of nonregenerative endothelial cells, generally shortening its lifespan (24). In addition, the true distribution of the hazard shape was unknown. We addressed this uncertainty by allowing for various distributions in the sensitivity analyses.

Third, in the absence of evidence regarding the share of patients that receive a favorably matched graft following HLA matching in a real-world setting, we had to draw on expert opinion to estimate an ICER. To give a conservative estimate for the effectiveness of HLA matching, the parameters we used for the analysis tended to (a) overestimate the share of patients that receive a favorably matching graft in the absence of HLA matching just by chance and (b) underestimate the share of patients that receives a favorably matched graft after HLA matching.

\section{CONCLUSIONS}

Favorable HLA matching based on a HLA Matchmaker appeared to be associated in everyday clinical practice not only with significantly longer rejection-free graft survival times but also with additional cost. Taking into account the peculiarities of grafts, our results suggest that the implementation of favorable HLA matching could in fact be viewed as cost-effective.

Given the innovative nature of the technology in question, however, we consider these results to be initial and recognize that further research is needed to evaluate the economic impact of the procedure. Future studies would preferably incorporate $\mathrm{RCT}$ data, and more comprehensive information regarding the share of patients receiving a favorably matched graft after HLA matching.

\section{CONTACT INFORMATION}

Michael Bäumler, PhD, Research Fellow, Department of Health Service Management, Ludwig-Maximilians-University, Munich, Germany

Leonie Sundmacher, PhD (sundmacher@bwl.lmu.de), Professor of Health Service Management, Department of Health Ser- vice Management, Ludwig-Maximilians-University, Munich, Germany

Thomas Reinhard, PhD, Chairman of Eye Clinic of the University Medical Center, Freiburg University, Germany

Daniel Böhringer, PhD, Professor of Ophthalmology, Eye Clinic of the University Medical Center, Freiburg University, Germany

\section{CONFLICTS OF INTEREST}

Thomas Reinhard and Daniel Böhringer received a grant from the Deutsche Forschungsgemeinschaft to perform a clinical trial in this field. The other authors report they have no potential conflicts of interest.

\section{REFERENCES}

1. Federal Health Monitoring of Germany - Gesundheitsberichterstattung des Bundes. Krankheitskosten, 2011. www.gbe-bund.de (accessed 2013 January 15, 2013).

2. Brown NN, Brown GC, Sharma S, Kistler J, Brown H. Utility values associated with blindness in an adult population. Br J Ophthalmol. 2001;85:327-331.

3. Armitage WJ, Tullo AB, Larkin DFP. The first successful full-thickness corneal transplant: A comment on Eduard Zirm's landmark paper of 1906. Br J Ophthalmol. 2006;90:1222-1223.

4. Coster DJ, Williams KA. The impact of corneal allograft rejection on the long- term outcome of corneal transplantation. Am J Ophthalmol. 2005;140:1112-1122.

5. Birnbaum F, Mayweg S, Reis A, et al. Mycophenolate mofetil (MMF) following penetrating high-risk keratoplasty: Long-term results of a prospective, randomised multicentre study. Eye (Lond). 2009;23:20632070.

6. Gomaa A, Comyn O, Liu C. Keratoprostheses in clinical practice - a review. Clin Exp Ophthalmol. 2010;38:211-224.

7. Duquesnoy RJ. Antibody-reactive epitope determination with HLAMatchmaker and its clinical applications. Tissue Antigens. 2011;77:525534.

8. Doxiadis II, Smits JM, Schreuder GM, et al. Association between specific HLA combinations and probability of kidney allograft loss: The taboo concept. Lancet. 1996;348:850-853.

9. Böhringer D, Daub F, Schwartzkopff J, et al. Operational postkeratoplasty graft tolerance due to differential HLAMatchmaker matching. Mol Vis. 2010;16:2362-2367.

10. Böhringer D, Reinhard S, Böhringer T, et al. Predicting time on the waiting list for HLA matched corneal grafts. Tissue Antigens. 2001;59:407411.

11. Böhringer D, Reinhard T, Sundmacher R. Systematische EDV-gestützte Erfassung der Nachuntersuchungsdaten von Keratoplastikpatienten: 10jähriger Erfahrungsbericht. Klin Monbl Augenheilkd. 2003;220:253256.

12. Reinhard T, Sundmacher R, Godehardt E, Heering P. Systemische Cyclosporin-A-Prophylaxe nach Keratoplastiken mit erhöhtem Risiko für Immunreaktionen als einzigem erhöhten Risikofaktor. Ophthalmologe. 1997;94:496-500.

13. Rubin DB. Matched sampling for causal effects. New York: Cambridge Press; 2006.

14. Bäumler M, Stargardt T, Schreyögg J, Busse R. Cost effectiveness of drugeluting stents in acute myocardial infarction patients in Germany: Results 
from administrative data using a propensity score-matching approach. Appl Health Econ Health Policy. 2012;10:235-248.

15. Austin PC. Some methods of propensity-score matching had superior performance to others: Results of an empirical investigation and Monte Carlo simulations. Biom J. 2009;51:171-184.

16. Austin PC. A critical appraisal of propensity-score matching in the medical literature between 1996 and 2003. Stat Med. 2008;27:20372049.

17. Reis A, Birnbaum F, Reinhard T. Systemic immunosuppressives after penetrating keratoplasty. Ophthalmologe. 2007;104:373-380.

18. Drummond MF, Schulpher MJ, Torrance GW, O’Brien JB, Stoddart GL. Methods for the economic evaluation of health care Programmes. 3rd ed. New York: Oxford University Press; 2005.

19. Allison PD. Survival analysis using SAS: A practical guide. 12th ed. Cary, NC: SAS Press; 2008.

20. Böhringer D, Maier P, Sundmacher R, Reinhard T. A cost calculation of an up-to-date eye Bank in Germany. In: Bredehorn-Mayr T, Duncker
GIW, Armitage WJ, eds. Developments in ophthalmology. Basel: Karger; 2009:120-124.

21. Birnbaum F, Böhringer D, Sokolovska Y, Sundmacher R, Reinhard T. Immunosuppression with cyclosporine A and mycophenolate mofetil after penetrating high-risk keratoplasty: A retrospective study. Transplantation. 2005;79:964-968.

22. Neymark N, Adriaenssen I, Gorlia T, Caleo S, Bolla M. Estimating survival gain for economic evaluations with survival time as principal endpoint: A cost-effectiveness analysis of adding early hormonal therapy to radiotherapy in patients with locally advanced prostate cancer. Health Econ. 2002;11:233-248.

23. Bonastre J, Chevalier J, Elias D, et al. Cost-effectiveness of intraperitoneal chemohyperthermia in the treatment of peritoneal carcinomatosis from colorectal cancer. Value Health. 2008;11:347-353.

24. Musch DC, Schwartz AE, Fitzgerald-Shelton K, Sugar A, Meyer RF. The effect of allograft rejection after penetrating keratoplasty on central endothelial cell density. Am J Ophthalmol. 1991;111:739-742. 